\title{
The influence of two-exciton correlations on the dynamics of parametric polariton amplification
}

\author{
Omar Di Stefano*, Salvatore Savasta, and Raffaello Girlanda \\ Istituto Nazionale per la Fisica della Materia INFM and Dipartimento di Fisica della Materia e \\ Tecnologie Fisiche Avanzate, Universitá di Messina Salita Sperone 31, 98166 Messina, Italy
}

\begin{abstract}
We present an overview of our results concerning the influence of two-pair continuum coherences on the transient nonlinear optical response of semiconductor microcavities. We show that the interplay between these 4-particle coherences and the nonperturbative light-matter interaction produces highly desirable almost decoherence-free exciton-exciton collisions on the lower polariton branch. This effect gives rise to the very different nonlinear absorption rates on the two polariton branches observed in many experiments and make possible to reach a very-high degree of amplification in samples with large Rabi splitting.
\end{abstract}

A semiconductor microcavity (SMC) is a photonic structure designed to enhance light-matter interactions. The dynamics and hence the resulting energy bands of these mixed quasiparticles are highly distorted with respect to those of bare excitons in embedded quantum wells (QWs) and cavity photons [1]. Very efficient amplification of polaritons has recently been reported [2]. Exciton systems are a good candidate as coherent amplifiers owing to the very large optical nonlinearities that they support when they are resonantly driven. Unfortunately $X X$ collisions are affected by decoherence producing excitation induced dephasing (EID). In amplifying systems the gain threshold is reached when the gain rate equals and then overcome the system decay rates increasing the system pumping. However if increasing the system pumping also increases decay rates (EID), efficient amplification would be prevented. Here we show that the capability of SMCs to alter the exciton dynamics during collisions producing almost decoherence-free collisions in the lower polariton branch is at the basis of the very large gains observed in these photon-exciton systems [3, 4].

The system that we investigate consists of one or more QWs grown inside a wavelength scale semiconductor planar Fabry-Perot. Moreover we focus on the case of input light beams with all the same circularly (e.g. $\sigma_{+}$) polarization, thus excluding the coherent excitation of bound two-pair coherences (biexcitons). We start from the Heisenberg equations for the exciton polarization and cavity field describing quantum optical effects and coherent nonlinear optics in SMCs [5], where the hierarchy of higher order density matrices has been closed by invoking the dynamics controlled truncation (DCT) scheme [6]. The variables describing two-pair transitions can be eliminated by formally inverting the corresponding equations of motion. This results in a memory kernel representation of the twopair transitions that is nonlocal in time and enters the equation of motion of single pair transition densities. The intracavity light-field is described within the quasi-mode scheme [5]. The time evolution of the coupled $1 s$ exciton $\left(P_{k}\right)$ and photon waves $\left(E_{k}\right)$ including finite duration of $X X$ collisions

\footnotetext{
* Corresponding author: e-mail: distef@ortica.unime.it
} 
$[7,4]$ obey the following set of coupled equations,

$$
\begin{aligned}
\frac{\partial}{\partial t} E_{k} & =-\left(\gamma_{c}+i \omega_{k}^{c}\right) E_{k}+i V P_{k}+t_{c} E_{k}^{\mathrm{in}} \\
\frac{\partial}{\partial t} P_{k} & =-\left(\gamma_{x}+i \omega_{x}\right) P_{k}+i V E_{k}-i \Omega_{k}^{\mathrm{NL}},
\end{aligned}
$$

where $\omega_{k}^{c}, \omega_{x}$, and $\gamma_{c}, \gamma_{x}$ are the energies and dephasing rates of cavity photons and QW excitons respectively. $E_{k}^{\text {in }}$ describes input light pulses (more precisely $\left|E_{k}^{\text {in }}\right|^{2}$ gives the input photon rate per unit area), $t_{c}$ determines the beam fraction passing the cavity mirror $\left(t_{c}=\sqrt{\gamma_{c}}\right.$ for a cavity with equal mirrors). The intracavity photon-field and the exciton field of a given mode $\boldsymbol{k}$ are coupled by $V$. The polariton splitting in SMCs can be increased by inserting a large number of QWs into the cavity ( $V=V_{1} \sqrt{N_{\text {eff }}}$, where $V_{1}$ is the exciton-photon coupling for $1 \mathrm{QW}$ and the effective number of QWs $N_{\text {eff }}$ depends on the number of wells inside the cavity and their spatial overlap with the cavity-mode). The relevant nonlinear source term, able to couple waves with different in-plane wave-vector $\boldsymbol{k}$, is given by $\Omega_{k}^{\mathrm{NL}}=\left(\Omega_{k}^{\mathrm{sat}}+\Omega_{k}^{X X}\right) / N_{\text {eff }}$, where the first term originates from the phase-space filling of the exciton transition,

$$
\Omega_{\boldsymbol{k}}^{\mathrm{sat}}=\frac{V}{n_{\mathrm{sat}}} \sum_{\boldsymbol{k}^{\prime} \boldsymbol{k}^{\prime \prime}} P_{\boldsymbol{q}}^{*} P_{\boldsymbol{k}^{\prime \prime}} E_{\boldsymbol{k}^{\prime}},
$$

being $n_{\text {sat }}=7 /\left(4 \pi a_{0}^{2}\right)$ the exciton saturation density $\left(\boldsymbol{k}^{\prime}, \boldsymbol{k}^{\prime \prime}\right.$, and $\boldsymbol{q}$ are tied by the momentum conservation relation $\left.\boldsymbol{k}+\boldsymbol{q}=\boldsymbol{k}^{\prime}+\boldsymbol{k}^{\prime \prime}\right) . a_{0}$ is the exciton Bohr radius determining the extension of the $1 s$ wavefunction: $\Phi_{X}(\boldsymbol{r})=2 \sqrt{2 / \pi a_{0}^{2}} \exp \left(-2 r / a_{0}\right) . \Omega_{\boldsymbol{k}}^{X X}$ is the Coulomb interaction term. It dominates the coherent $X X$ coupling and for co-circularly polarized waves can be written as

$$
\Omega_{\boldsymbol{k}}^{X X}=\sum_{\boldsymbol{k}^{\prime} \boldsymbol{k}^{\prime \prime \prime}} V_{X X} P_{\boldsymbol{q}}^{*}(t) P_{\boldsymbol{k}^{\prime \prime}}(t) P_{\boldsymbol{k}^{\prime}}(t)-i P_{\boldsymbol{q}}^{*}(t) \int_{-\infty}^{t} F\left(t-t^{\prime}\right) P_{\boldsymbol{k}^{\prime \prime}}\left(t^{\prime}\right) P_{\boldsymbol{k}^{\prime}}\left(t^{\prime}\right) \mathrm{d} t^{\prime}
$$

$\Omega_{k}^{X X}$ includes the instantaneous mean-field $X X$ interaction term $V_{X X} \simeq 1.52 E_{b} a_{0}^{2}\left(E_{b}\right.$ is the exciton binding energy) plus a non instantaneous term originating from 4-particle correlations. This coherent memory can be interpreted as a non-markovian process involving the 2-particle (excitons) polarization waves interacting with a bath of 4-particle correlations $[8,9]$. The memory kernel $F(\tau)$ can be written in the following form,

$$
F(\tau)=\Theta(\tau) \sum_{m}\left|\left\langle 0|\hat{D}| E_{m}\right\rangle\right|^{2} \mathrm{e}^{-\left(i \omega_{m}+\Gamma\right) \tau},
$$

where $\Theta(\tau)$ is the step function, $\hat{D}=\left[\hat{B},\left[\hat{B}, \hat{V}_{C}\right]\right]$ ( $\hat{B}$ is the exciton destruction operator, while $\hat{V}_{C}$ is the Coulomb interaction operator), and $\left|E_{m}\right\rangle$ and $\omega_{m}$ describe the eigenstates and the corresponding continuous spectrum of energies of the 4-particle Hilbert subspace [7, 9, 10]. $\Gamma$ describes the dephasing of two pair coherences (in the following we will use $\Gamma=2 \gamma_{x}$ ). We calculated $F(\tau)$ for QW excitons following a recent microscopic approach [11,12] based on the T-matrix (Fig. 1). By this approach the 4-particle states $\left|E_{m}\right\rangle$ are expanded in terms of exciton-pairs on the $1 s$ parabola. The inset in Fig. 1 displays $F(\tau)$ calculated for a $7 \mathrm{~nm}$ GaAs QW with binding energy $E_{b}=13.5 \mathrm{meV}$. Figure 1 displays $\mathcal{F}(\omega)$ that is the Fourier transform of $F(\tau)$ plus the mean-field term $V_{X X}$ :

$$
\mathcal{F}(\omega)=V_{X X}+\int_{-\infty}^{\infty} \mathrm{d} t F(t) \mathrm{e}^{i \omega t} .
$$

The imaginary part of $\mathcal{F}(\omega)$ reflects the density of states of 2-exciton pair coherences.

Equation (1) clearly displays the structure of the polariton-polariton scattering mechanism: $\Omega_{k}^{\mathrm{NL}}$ couples exciton waves belonging to different $\boldsymbol{k}$ modes. We also observe that the strong exciton-photon coupling does not modify the memory kernel $F(\tau)$ as a consequence of the fact that 4-particle correlations do not couple to cavity photons $[5,7,12]$. However cavity effects are able to alter the phase dynamics of the 2-particle polarization waves $P_{\boldsymbol{k}}$ during collisions, i.e. on a timescale shorter than the 


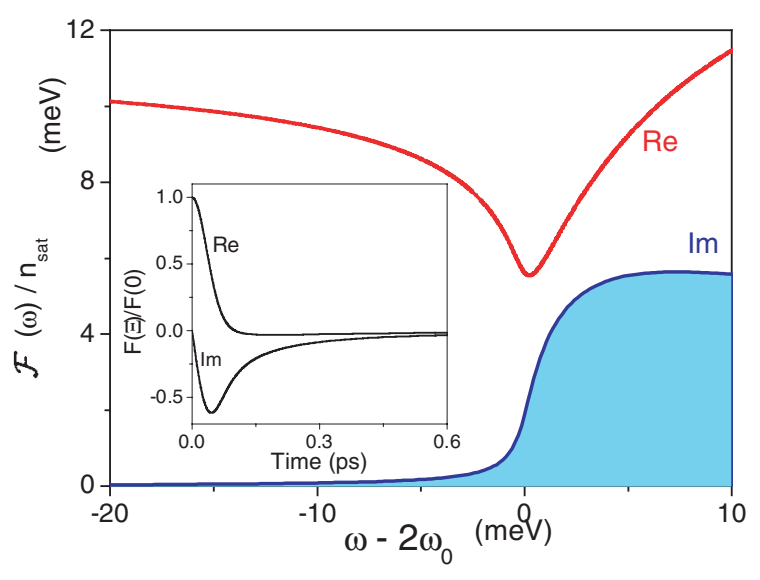

Fig. 1 Energy dependence of the effective excitonexciton scattering potential. It has been calculated for a GaAs QW $7 \mathrm{~nm}$ wide with exciton binding energy $13.5 \mathrm{meV}$ and using as dephasing rate of the 4-particle states $\Gamma=2 \gamma_{x}=0.58 \mathrm{meV}$. The tail of $\operatorname{Im}(\mathcal{F}(\omega))$ at negative detuning $\left(\omega<2 \omega_{0}\right)$ is produced by $\Gamma$ and vanishes for $\Gamma \rightarrow 0$. The inset shows the dynamics of the memory kernel $F(\tau)$. b Feynman diagrams describing the effective excitonexciton interaction as modified by polariton effects.

decay time of the memory kernel $F(\tau)$. In particular the phase of 2-particle polarization waves in SMCs oscillates with a frequency (fixed by the polariton dispersion relations) modified respect to that of excitons in bare QWs. This fact can produce a deep modification of the integral in Eq. (3). This mechanism can be more clearly understood simplifying the integral in Eq. (3). Inserting Eq. (4) into Eq. (3), the resulting integral term resembles the corresponding term determining the spontaneous decay of a two-level transition [13]. It is possible to simplify the integral in Eq. (3) following this analogy. We can write the polarization densities as $P_{k}(t)=X_{k}(t) \mathrm{e}^{-i \omega_{k} t}$ and can assume that the amplitudes $X_{k}(t)$ are slowly varying functions of time as compared to the ultrafast decay time of the memory function $F(\tau)$ (see the inset in Fig. 1). Then, adopting the well-known Weisskopf-Wigner approximation used to analyze the spontaneous emission between two atomic levels, the dominant $X X$ interaction term for the signal $(0)$ and idler $(2 \boldsymbol{k})$ modes can be written as [7]

$$
\Omega_{0(2 \boldsymbol{k})}^{X X}=2 \mathcal{F}\left(\omega_{0(2 k)}+\omega_{k}\right)\left|P_{\boldsymbol{k}}(t)\right|^{2} P_{0(2 k)}(t)+\mathcal{F}\left(2 \omega_{k}\right) P_{2 \boldsymbol{k}(0)}^{*}(t) P_{\boldsymbol{k}}^{2}(t) .
$$

The spontaneous emission rate of atoms, according to the Weisskopf-Wigner theory depends on the optical density of states calculated at the transition energy. Analogously Eq. (5) shows that the $X X$ scattering rates depend on the spectral density of two pair transitions calculated at the energy of the colliding polariton pairs. The first term (arising from self-scattering of polariton pairs) produces a blue-shift of the polariton resonance and introduces an intensity-dependent dephasing mechanism. The second term provides the coupling mechanism able to transfer polaritons from the pump to the signal and idler modes. This term describes the scattering of a polariton pair ( 2 pump polaritons) into a different pair (the signal plus the idler) with pair energy and momentum conservation. An analogous expression can be derived for the exciton-exciton interaction of the pump mode. This WeisskopfWigner description of 4-particle correlation effects in SMCs sets out the relevance of polariton pairs in the scattering process. The obtained 4-particle spectral density (see Fig. 1) displays strong variations within the spectral region of interest around $2 \omega_{0}$. In particular, moving towards the low energy region, the dispersive part $\operatorname{Re}(\mathrm{F})$ increases while the absorptive part $\operatorname{Im}(\mathcal{F})$ that contrasts gain goes to zero. As a consequence, the exciton-exciton scattering rates in SMCs differ significantly from those of resonantly excited excitons in bare QWs. Mean-field calculations do not take into account this difference. We observe that (i) these spectral variations of $\mathcal{F}(\omega)$ origins from the finite duration of the $X X$ interaction (instantaneous $X X$ collisions would have implied the absence of spectral variations of $\mathcal{F}(\omega)$ ). (ii) Owing to the spectral behavior of $\mathcal{F}(\omega)$, changing the polariton resonances, e.g. by changing the polariton splitting or varying the detuning (the energy difference between the cavity and exciton resonances) it is possible to alter profoundly the scattering rates.

The Weisskopf-Wigner approximation just provided a useful guideline to understand the experimental findings. In the following we present numerical results obtained solving the system of 6 integro-differential equations for three modes: the signal (at normal incidence), the pump ( $\boldsymbol{k}$ generate by 

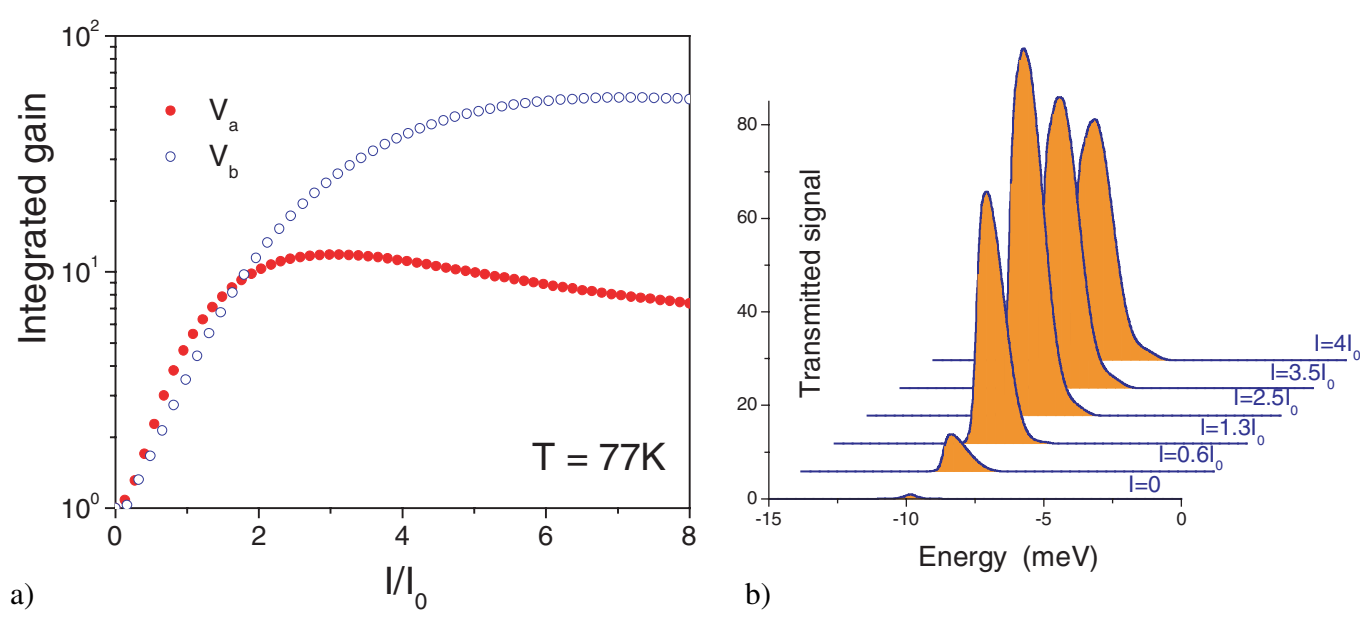

Fig. 2 a) Power dependence of the integrated gain calculated for two GaAlAs based samples with splitting $2 V_{a}=10.6 \mathrm{meV}$ and $2 V_{b}=15 \mathrm{meV} . I_{0}=10^{13}$ photons per $\mathrm{cm}^{2}$ per pulse. The exciting light pulses have $250 \mathrm{fs}$ duration. Material parameters of the two samples are coincident except for $N_{\text {eff. }}$ The decay rate of cavity photons through the mirrors $\gamma_{c}=0.25 \mathrm{meV}$ is that of GaAlAs structures considered in Ref. [3]; b) gain spectra for different pump excitation densities obtained for the sample a at $T=10 \mathrm{~K}$.

light seeding the cavity at the magic angle of incidence such that $\left.2 \Omega_{k}=\Omega_{0}+\Omega_{2 k}\right)$, and the idler $(2 \mathrm{k}$ ). The microscopic theory of polariton parametric amplification here presented allows a fully quantitative analysis of recent measurements. The gain curves versus the input pump power calculated for GaAlAsbased samples with splitting $2 V_{a}=10.6 \mathrm{meV}\left(N_{\text {eff }}=4\right)$ and $2 V_{b}=15 \mathrm{meV}\left(N_{\text {eff }}=8\right)$ (Fig. 2a), fully confirm the analysis based on the Weisskopf-Wigner theory. The calculated gain is defined as the total light intensity transmitted in the signal direction divided by the intensity transmitted in the absence of the pump beam. Figure 2a) shows that an increase of less then a factor $1 / 3$ in the polariton splitting produces an increase of the maximum achievable gain at $T=77 \mathrm{~K}$ of six time in agreement with experimental results [3]. This result shows how the increase of the exciton-photon coupling favours high-temperature operation. The power dependence of gain shows an almost exponential growth and then saturates at high powers. The saturation of gain is mainly determined by the nonlinear absorption that is most relevant for the idler beam according to Eq. (4) the idler nonlinear absorption is determined by $\operatorname{Im} \mathcal{F}\left(\omega_{2 k}+\omega_{k}\right)$. We observe that the increase of the idler nonlinear absorbance is highly superlinear because the increase of the pump power produces both a direct increase of the exciton density and an increase of $\operatorname{Im} \mathcal{F}\left(\omega_{2 k}+\omega_{k}\right)$ as a consequence of the blue shift of the polariton-pair resonance $\omega_{2 k}+\omega_{k}$ induced by $\operatorname{Re} \mathcal{F}$. Figure $2 b$ ) shows gain spectra for different pump excitation densities obtained for the sample a at $T=10 \mathrm{~K}$. The spectra clearly displays the effects of blue-shift, amplification and EID. In particular notice the broadening of the peaks produced by the EID at the highest pump densities.

The results here shown clarifies the origin of the great enhancement of parametric gain observed when increasing the polariton splitting. Observing that the wave-like nature of excitations in solids tells us that collisions between excitons are nothing but interference phenomena we are now able to affirm that the strong-coupling regime of SMCs, altering the phase of the interacting waves, provides a means to control the interference process. This coherent control of exciton-exciton interactions is able to produce highly desirable almost decoherence-free collisions that make possible to reach a very-high degree of amplification. Almost decoherence-free exciton-exciton interactions and hence structures with large polariton splitting are expected to be essential to reach room temperature operation. Moreover the availability of decoherence-free collisions appears crucial for the quantum control and manipulation of the polariton wavefunction inside the cavity. We believe that the possible control of exciton-exciton collisions opens new perspectives for the realization of entangled collective polariton states for quantum information and computation. 


\section{References}

[1] C. Weisbuch et al., Phys. Rev. Lett. 69, 3314 (1992).

[2] P. G. Savvidis, et al., Phys. Rev. Lett. 84, 1547 (2000).

[3] M. Saba et al., Nature 414, 731 (2002).

[4] S. Savasta, O. Di Stefano, and R. Girlanda, Phys. Rev. Lett. 90, 096403 (2003).

[5] S. Savasta and R. Girlanda, Phys. Rev. Lett. 77, 4736 (1996).

[6] V. M. Axt and S. Mukamel, Rev. Mod. Phys. 70, 145 (1998).

[7] S. Savasta et al., Phys. Rev. B 64, 073306 (2001).

[8] D. S. Chemla and J. Shah, Nature. 411, 549 (2001).

[9] T. Östreich et al., Phys. Rev. Lett. 74, 4698 (1995).

[10] S. Savasta and R. Girlanda, Phys. Rev. B 59, 15409 (1999).

[11] R. Takayama et al., Eur. Phys. J. B 25, 445-462 (2002).

[12] N. H. Kwong et al., Phys. Rev. Lett. 87, 027402 (2001).

[13] See e.g. M. O. Scully and M. Zubairy, Quantum Optics, (Cambridge University Press 1997). 